Proyecciones Journal of Mathematics Vol. 35, $\mathrm{N}^{o}$ 1, pp. 33-44, March 2016.

Universidad Católica del Norte

Antofagasta - Chile

\title{
Equi independent equitable dominating sets in graphs
}

\author{
S. K. Vaidya \\ Saurashtra University, India \\ and \\ $N J$ Kothari \\ L. E. College, India
}

Received : March 2015. Accepted : December 2015

\begin{abstract}
We introduce the concept of an equi independent equitable dominating set and define equi independent equitable domination number. We also investigate the graph families whose equi independent equitable domination number and equitable domination number are same.
\end{abstract}

Keywords : Equi independent equitable domination number, equitable domination number, domination number.

AMS subject classification number : 05C69;05C38. 


\section{Introduction}

In any group of people equality of status, power, wealth etc. is desirable and it is also a remarkable feature of constitution of a democratic nation. Keeping this idea in to center Sampathkumar has introduced the concept of equitable domination. On the other hand some social status like marital relationship must be enjoyed individually. Also the information and password security for financial matters must be independent in order to retain uniqueness. Such issues have motivated the concept of independent domination which was formalized by Berge [1] and Ore [6] while the independent domination and the notation $i(G)$ were introduced by Cockayne and Hedetniemi in $[2,3]$. A survey on independent domination can be found in Goddard and Henning [4].

Is it possible to think about a set of people who can enjoy both the characteristics namely, equality and independence simultaneously? In attempt to answer this question, the idea of equitable independent equitable domination was conceived by Swaminathan and Dharmalingam [7]. We formalize this concept and term as an equi independent equitable domination. We provide some prerequisites for the initiation of new concept.

In this paper, the term graph we mean simple, finite, connected and undirected graph $G=(V(G), E(G))$. For terminology and notation not defined here we follow West [11] and Haynes et al. [5]. For every vertex $v \in$ $V(G)$, the open neighbourhood set $N(v)$ is the set of all vertices adjacent to $v$ in $G$. That is, $N(v)=\{u \in V(G) / u v \in E(G)\}$. The closed neighbourhood set $N[v]$ of $v$ is defined as $N[v]=N(v) \cup\{v\}$. A set $D \subseteq V(G)$ is called a dominating set if every vertex in $V(G)-D$ is adjacent to at least one vertex in $D$. The domination number $\gamma(G)$ is the minimum cardinality of a dominating set of $G$. A subset $D$ of $V(G)$ is called an equitable dominating set if for every $v \in V-D$ there exists a vertex $u \in D$ such that $u v \in E(G)$ and $|\operatorname{deg}(u)-\operatorname{deg}(v)| \leq 1$. The minimum cardinality of such a dominating set is denoted by $\gamma^{e}$ and is called the equitable domination number of $G$. A vertex $u \in V(G)$ is said to be degree equitable with a vertex $v \in V(G)$ if $|\operatorname{deg}(u)-\operatorname{deg}(v)| \leq 1$. An equitable dominating set $D$ is said to be a minimal equitable dominating set if no proper subset of $D$ is an equitable dominating set.

Swaminathan and Dharmalingam [7] have derived following necessary and sufficient condition for minimal equitable dominating set.

Theorem 1.1. An equitable dominating set $D$ is minimal if and only if for every vertex $u \in D$ one of the following holds. 
(i) Either $N(u) \cap D=\emptyset$ or $|\operatorname{deg}(v)-\operatorname{deg}(u)| \geq 2$ for all $N(u) \cap D$.

(ii) There exists a vertex $v \in V(G)-D$ such that $N(v) \cap D=\{u\}$ and $|\operatorname{deg}(v)-\operatorname{deg}(u)| \leq 1$.

A vertex $v \in V(G)$ is called equitable isolates if $|d(v)-d(u)| \geq 2$ for every $u \in N(v)$. Note that equitable isolates must belongs to any equitable dominating set of graph $G$. Obviously isolated vertices are equitable isolated vertices. Hence $I_{s} \subseteq I_{e} \subseteq D$ for every equitable dominating set $D$, where $I_{s}, I_{e}$ are the set of all isolated vertices and set of all equitable isolates of $G$ respectively.

Theorem 1.2. [7] A graph $G$ has a unique minimal equitable dominating set if and only if the set of all equitable isolates forms an equitable dominating set.

The equitable neighbourhood of $v$ denoted by $N^{e}(v)$ is defined as $N^{e}(v)=$ $\{u \in V(G) / u \in N(v),|\operatorname{deg}(v)-\operatorname{deg}(u)| \leq 1\}$. The cardinality of $N^{e}(v)$ is denoted by $\operatorname{deg}_{G}^{e}(v)$. The maximum and minimum equitable degree of graph $G$ are denoted by $\Delta^{e}(G)$ and $\delta^{e}(G)$ respectively which are defined as $\Delta^{e}(G)=\max _{v \in V(G)}\left|N^{e}(v)\right|, \delta^{e}(G)=\min _{v \in V(G)}\left|N^{e}(v)\right|$.

Remark 1.3. $\Delta^{e}(G) \leq \Delta(G)$

Remark 1.4. For a connected graph $G, \delta^{e}(G)$ may be zero. For star graph $K_{1, n}, \Delta^{e}(G)=\delta^{e}(G)=0$.

Swaminathan and Dharamlingam [7] have also introduced the concept of equitable independent set. According to them a subset $S$ of $V(G)$ is called an equitable independent set if for any $u \in S, v \notin N^{e}(u)$ for all $v \in S-\{u\}$. The maximum cardinality of an equitable independent set is denoted by $\beta^{e}(G)$.

Remark 1.5. Every independent set is an equitable independent set.

Theorem 1.6. [7] Let $S$ be a maximal equitable independent set. Then $S$ is a minimal equitable dominating set.

Definition 1.7. The helm $H_{n}$ is the graph obtained from a wheel $W_{n}$ by attaching a pendant edge to each of the rim vertex.

Definition 1.8. The closed helm $\mathrm{CH}_{n}$ is the graph obtained from helm $H_{n}$ by joining each pendant vertex to form a cycle. 
An equitable dominating set $D$ is said to be equi independent equitable dominating set if it is also equitable independent set. The minimum cardinality of an equi independent equitable dominating is called equi independent equitable domination number which denoted by $i^{e}$.

Illustration 1.9. In Figure $1, D=\left\{v_{1}, v_{2}, v_{3}, v_{5}, v_{7}, v_{9}\right\}$ is equitable independent set as well as equitable dominating set for graph $G$.

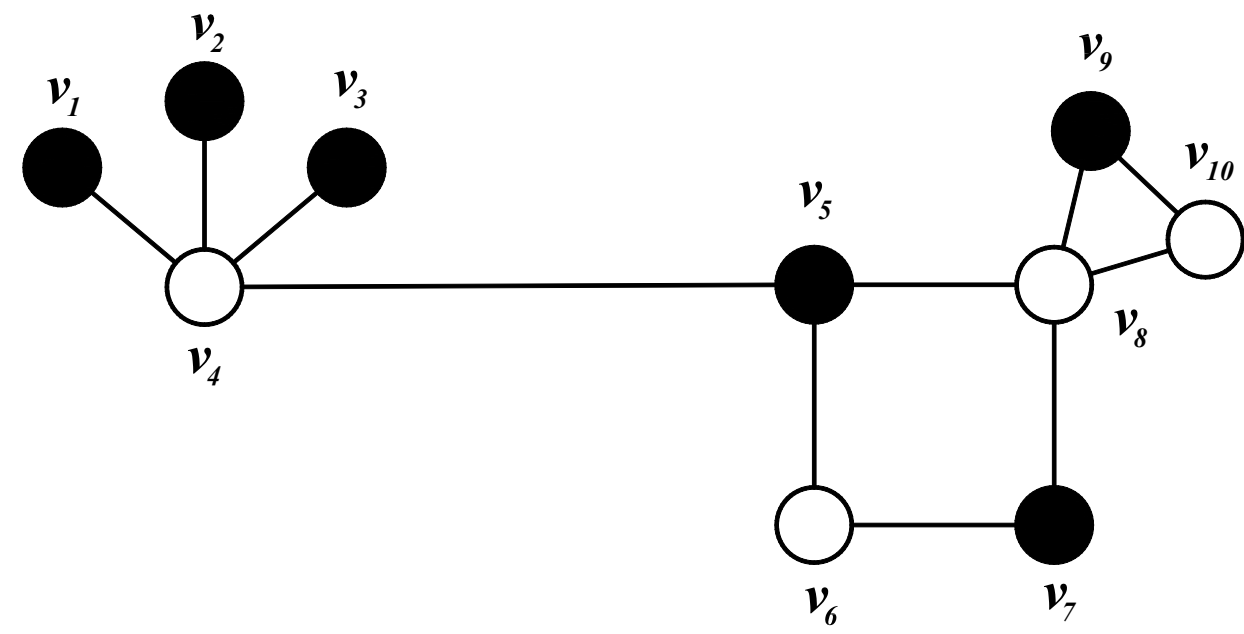

Figure 1: Equi independent equitable dominating set of graph $G$

The equi independent equitable domination number have been investigated for various graph families in $[8,9,10]$.

\section{Main Results}

Theorem 2.1. Let $G$ be a graph in which all the vertices are equitable isolates then $i^{e}(G)=\gamma^{e}(G)=n$. 
Proof. Let $G$ be a graph in which all the vertices are equitable isolates then each vertex can equitably dominate it self only. While every pair of vertices $u, v \in V(G)$ are equitably non adjacent. Which implies that $V(G)$ is $\gamma^{e}$-set of $G$ and maximal independent set. Hence $i^{e}(G)=\gamma^{e}(G)=n$.

We define $n\left(e_{i}\right)$ as number of equitable isolates in graph $G$. The following theorem gives the lower bound for an equi independent equitable dominating set.

Theorem 2.2. $i^{e}(G) \geq \gamma^{e}(G) \geq n\left(e_{i}\right)+i^{e}\left(G-I_{e}\right)$.

Proof. Let $G$ be a graph. If graph $G$ have equitable isolates then they must be in any equi independent equitable dominating set. Therefore any equi independent equitable dominating set must contain equitable isolates and $i^{e}$-set of $G-I_{e}$. While if graph $G$ is without equitable isolates then $n\left(e_{i}\right)=0$. Hence $i^{e}(G) \geq \gamma^{e}(G) \geq n\left(e_{i}\right)+i^{e}\left(G-I_{e}\right)$.

Remark 2.3. The graph shown in figure 1 achieves the lower bound of the above theorem.

Theorem 2.4. $i^{e}\left(P_{n}\right)=\gamma^{e}\left(P_{n}\right)=\gamma\left(P_{n}\right)$.

Proof. Let $D$ be any $\gamma$-set of path $P_{n}$. Then it is an equi independent equitable dominating set of $P_{n}$ as $d\left(v_{i}\right)=1$ or 2 for every $i$. Also it is equitable independent set as any $\gamma$-set of $P_{n}$ is independent set. Hence $i^{e}\left(P_{n}\right)=\gamma^{e}\left(P_{n}\right)=\gamma\left(P_{n}\right)$.

Theorem 2.5. $i^{e}\left(C_{n}\right)=\gamma^{e}\left(C_{n}\right)=\gamma\left(C_{n}\right)$.

Proof. Let $D$ be a $\gamma$-set of $C_{n}$. Since $C_{n}$ is 2-regular graph, any $\gamma$-set of $C_{n}$ is an equitable dominating set of $C_{n}$. Also any $\gamma$-set of $C_{n}$ is an independent set. Hence $i^{e}\left(P_{n}\right)=\gamma^{e}\left(P_{n}\right)=\gamma\left(P_{n}\right)$.

Theorem 2.6. $i^{e}\left(K_{n}\right)=\gamma^{e}\left(K_{n}\right)=\gamma\left(K_{n}\right)$.

Proof. Any singleton subset of $V\left(K_{n}\right)$ form equitable independent as well as equitable dominating set. Hence $i^{e}\left(K_{n}\right)=\gamma^{e}\left(K_{n}\right)=\gamma\left(K_{n}\right)$.

Theorem 2.7. $i^{e}\left(W_{n}\right)=\gamma^{e}\left(W_{n}\right)=\gamma\left(W_{n}\right)=1$ for $n=3,4$. 
Proof. Let $v_{1}, v_{2}, \ldots, v_{i}$ be the rim vertices of $W_{i}$ and $v$ be a apex vertex of $W_{i}$ where $i=3,4$. Observe that $D=\{v\}$ is an equitable independent set as being singleton set and it is an equitable dominating set as it is apex vertex with $\left|d(v)-d\left(v_{i}\right)\right|=1$. Hence $i^{e}\left(W_{n}\right)=\gamma^{e}\left(W_{n}\right)=\gamma\left(W_{n}\right)=1$.

Theorem 2.8. $i^{e}\left(W_{n}\right)=\gamma^{e}\left(W_{n}\right)=\left\lceil\frac{n}{3}\right\rceil+1$ for $n>4$.

Proof. Let $v_{1}, v_{2}, \ldots, v_{n}$ be the rim vertices, $v$ be a apex vertex of $W_{n}$. Observe that apex vertex $v$ is equitable isolates. Therefore $\gamma^{e}\left(W_{n}\right) \geq$ $\gamma^{e}\left(C_{n}\right)+1$. Let $S=\gamma^{e}$-set of $C_{n}$ and $D=S \cup\{v\}$ with $|D|=\gamma^{e}\left(C_{n}\right)+$ 1. Observe that $D$ is an equitable dominating set and it is an equitable independent set as it contain equitable isolates vertex $v$ and $\gamma^{e}$-set of $C_{n}$, which is maximal equitable independent set of $C_{n}$. Hence $i^{e}\left(W_{n}\right)=$ $\gamma^{e}\left(W_{n}\right)=\left\lceil\frac{n}{3}\right\rceil+1$.

Theorem 2.9. $i^{e}\left(K_{m, n}\right)=\gamma^{e}\left(K_{m, n}\right)$.

Proof. Let $K_{m, n}$ be the complete bipartite graph with $m$ vertices in one partition say $V_{1}$ and $n$ vertices in other partition say $V_{2}$. Therefore $V\left(K_{m, n}\right)=V_{1} \cup V_{2}$. Observe that $\operatorname{deg}(u)=\left\{\begin{array}{cl}n & \text { if } u \in V_{1} \\ m & \text { if } u \in V_{2}\end{array}\right.$

Let $D$ be any $\gamma^{e}$-set of $K_{m, n}$.

Case 1: $|m-n| \leq 1$.

In this case $\gamma^{e}\left(K_{m, n}\right)=2$ with $D=\left\{u, v / u \in V_{1}, v \in V_{2}\right\}$. Therefore $D$ is an independent set which implies that $D$ is an equitable independent set. Therefore $D$ is an equi independent equitable dominating set. Hence $i^{e}\left(K_{m, n}\right)=\gamma^{e}\left(K_{m}, n\right)$.

Case 2: $|m-n|>2$

In this case $\gamma^{e}\left(k_{m, n}\right)=m+n$. Therefore $\gamma^{e}$-set of $K_{m, n}=i^{e}$-set of $K_{m, n}$ $=V\left(K_{m, n}\right)$, as all the vertices are equitable isolates. Hence $i^{e}\left(K_{m, n}\right)=$ $\gamma^{e}\left(K_{m}, n\right)$.

Theorem 2.10. $i^{e}\left(H_{n}\right)= \begin{cases}\gamma^{e}\left(H_{n}\right)=n+1 & \text { for } n=3,4,5 \\ \gamma^{e}\left(C_{n}\right)+n+1 & \text { for } n \geq 6\end{cases}$ 
Proof. Let $v_{1}, v_{2}, \ldots, v_{n}$ be rim vertices, $u_{1}, u_{2}, \ldots, u_{n}$ be the pendant vertices and $v$ be the apex vertex of helm $H_{n}$.

Case 1: $n=3,4,5$

In this case vertices $u_{1}, u_{2}, \ldots, u_{i}$ are equitable isolates for $i=3,4$. Therefore they must belongs to any equitable dominating set. Let

$D=\left\{u_{1}, u_{2}, \ldots, u_{i}, v\right\}$ for $i=3,4$. Observe that $D$ is equitable dominating set as apex vertex $v$ equitably dominate rim vertices $v_{1}, v_{2}, \ldots, v_{i}$ for $i=3,4$. Also $D$ is independent set which implies that $D$ is equitable independent set. Therefore $D$ is an equi independent equitable dominating set. Hence $i^{e}\left(H_{n}\right)=\gamma^{e}\left(H_{n}\right)=n+1$.

Case 2: $n \geq 6$

In this case $d\left(u_{i}\right)=1, d\left(v_{i}\right)=4$ and $d(v)=n$. This implies that pendant vertices $u_{1}, u_{2}, \ldots, u_{n}$ and apex vertex $v$ are equitable isolates. Therefore they must belongs to any equitable dominating set. Also subgraph induced by $V\left(H_{n}\right)-\left\{u_{1}, u_{2}, \ldots, u_{n}, v\right\}$ is cycle $C_{n}$. Which implies that $\gamma^{e}\left(H_{n}\right) \geq$ $\gamma^{e}\left(C_{n}\right)+n+1$. Let $S$ be a $\gamma^{e}$-set of $C_{n}$ and $D=\left\{u_{1}, u_{2}, \ldots, u_{n}\right\} \cup S$. Observe that $D$ is an equitable dominating set as all pendant vertices and apex vertex are in $D$, while rim vertices are equitably dominate by set $S$. Also $D$ is an equitable independent set. Hence $D$ is an equi independent equitable dominating set and $i^{e}\left(H_{n}\right)=\gamma^{e}\left(H_{n}\right)+n+1$.

Theorem 2.11. $i^{e}\left(C H_{n}\right)= \begin{cases}\gamma^{e}\left(C H_{n}\right)=2 & \text { for } n=3,4 \\ \gamma^{e}\left(C H_{n}\right)=3 & \text { for } n=5\end{cases}$

Proof. Let $v$ be apex vertex with $d(v)=n, v_{1}, v_{2}, \ldots, v_{n}$ be the vertices of degree 4 and $u_{1}, u_{2}, \ldots, u_{n}$ be vertices of degree 3 of $\mathrm{CH}_{n}$.

Case 1: $n=3,4$

In this case $\left|V\left(\mathrm{CH}_{3}\right)\right|=2 n+1$ and none of the vertex having degree $2 n$. Therefore $\gamma^{e}\left(\mathrm{CH}_{n}\right)=\gamma\left(\mathrm{CH}_{n}\right)>1$. Consider $D=\left\{v_{1}, u_{3}\right\}$ with $|D|=2$. Then $N^{e}[D]=V\left(C H_{n}\right)$. Therefore $D$ is an equitable dominating set of $C H_{n}$. Also vertices $v_{1}$ and $u_{3}$ are not adjacent to each other. This implies that set $D$ is an independent set as well as an equitable independent set of $\mathrm{CH}_{n}$. Thus $D$ is an equi independent equitable dominating set of $\mathrm{CH}_{n}$ and $i^{e}\left(\mathrm{CH}_{n}\right)=\gamma^{e}\left(\mathrm{CH}_{n}\right)=2$.

Case 2: $n=5$

In this case $\left|V\left(\mathrm{CH}_{5}\right)\right|=11$. and none of the vertex having degree 10 . 
Therefore $\gamma^{e}\left(\mathrm{CH}_{5}\right)=\gamma\left(\mathrm{CH}_{5}\right)>1$. Consider $D=\left\{v, u_{1}, u_{3}\right\}$ with $|D|=3$. Then $N^{e}[D]=V\left(C_{5}\right)$. Therefore $D$ is an equitable dominating set of $\mathrm{CH}_{5}$. Also vertices $v, u_{1}, u_{3}$ are not adjacent to each other. This implies that set $D$ is an independent set as well as an equitable independent set of $\mathrm{CH}_{5}$. Thus $\mathrm{D}$ is an equi independent equitable dominating set of $\mathrm{CH}_{5}$ and $i^{e}\left(\mathrm{CH}_{5}\right)=\gamma^{e}\left(\mathrm{CH}_{5}\right)=3$.

\section{Theorem 2.12.}

$$
i^{e}\left(C H_{n}\right)= \begin{cases}\gamma^{e}\left(C H_{n}\right)= \begin{cases}2\left\lfloor\frac{n}{4}\right\rfloor+3 & \text { for } n \equiv 2,3(\bmod 4), n \neq 3 \\ \frac{n}{2}+1 & \text { for } n \equiv 0(\bmod 4), n \neq \equiv 4\end{cases} \\ \gamma^{e}\left(C H_{n}\right)+1=\left(2\left\lfloor\frac{n}{4}\right\rfloor+2\right)+1 \text { for } n \equiv 1(\bmod 4), n \neq 5\end{cases}
$$

Proof. To prove this result we continue with the terminology and notations used in Theorem 2.11. Observe that apex vertex $v$ is an equitable isolates. while other vertices are equitably adjacent to each other.

Case 1: $n \equiv 0(\bmod 4)$

Here $N^{e}\left(v_{4 i+1}\right)=\left\{v_{4 i}, v_{4 i+2}, u_{4 i+1}\right\}$ and $N^{e}\left(u_{4 i+3}\right)=\left\{u_{4 i+2}, u_{4 i}, v_{4 i+3}\right\}$. Let $D=\left\{v, v_{4 i+1}, u_{4 i+3}\right\}$ where $0 \leq i \leq \frac{n}{4}-1$ with $|D|=\frac{n}{2}+1$. Here $v$ is an equitable isolate and vertices $v_{4 i+1}, u_{4 i+3}$ are not equitably adjacent to each other. Therefore $D$ is an equitable independent set. Other hand $N^{e}(v) \cup N^{e}\left(v_{4 i+1}\right) \cup N^{e}\left(u_{4 i+3}\right)=V\left(C H_{n}\right)$, where $0 \leq i \leq \frac{n}{4}-1$. Therefore $D$ is an equitable dominating set. Hence $D$ is an equi independent equitable dominating set and $i^{e}\left(\mathrm{CH}_{n}\right)=\gamma^{e}\left(\mathrm{CH}_{n}\right)=\frac{n}{2}+1$.

Case 2: $n \equiv 1(\bmod 4)$

Here $N^{e}\left(v_{4 i+1}\right)=\left\{v_{4 i}, v_{4 i+2}, u_{4 j+1}\right\}$ and $N^{e}\left(u_{4 j+3}\right)=\left\{u_{4 j+2}, u_{4 j}, v_{4 i+3}\right\}$. Let $D=\left\{v, v_{4 i+1}, u_{4 j+3}, v_{n-1}, u_{n}\right\}$ where $0 \leq i \leq\left\lfloor\frac{n}{4}\right\rfloor, 0 \leq j<\left\lfloor\frac{n}{4}\right\rfloor$ with $|D|=2\left\lfloor\frac{n}{4}\right\rfloor+3$. Here vertex $v$ is an equitable isolate and vertices $v_{4 i+1}, u_{4 j+3}, v_{n-1}, u_{n}$ are not equitably adjacent to each other. Therefore $D$ is an equitable independent set. Also $N^{e}(v) \cup N^{e}\left(v_{4 i+1}\right) \cup N^{e}\left(u_{4 j+3}\right)=$ $V\left(C H_{n}\right)$, where $0 \leq i \leq\left\lfloor\frac{n}{4}\right\rfloor, 0 \leq j<\left\lfloor\frac{n}{4}\right\rfloor$. Therefore $D$ is an equitable dominating set. Hence $D$ is an equi independent equitable dominating set and $i^{e}\left(\mathrm{CH}_{n}\right)=\gamma^{e}\left(\mathrm{CH}_{n}\right)+1=\left(2\left\lfloor\frac{n}{4}\right\rfloor+2\right)+1$.

Case 3: $n \equiv 2(\bmod 4)$

Here $N^{e}\left(v_{4 i+1}\right)=\left\{v_{4 i}, v_{4 i+2}, u_{4 j+1}\right\}$ and $N^{e}\left(u_{4 j+3}\right)=\left\{u_{4 j+2}, u_{4 j}, v_{4 i+3}\right\}$. Let $D=\left\{v, v_{4 i+1}, u_{4 j+3}, u_{n}\right\}$ where $0 \leq i \leq\left\lfloor\frac{n}{4}\right\rfloor, 0 \leq j<\left\lfloor\frac{n}{4}\right\rfloor$ with $|D|=$ $2\left\lfloor\frac{n}{4}\right\rfloor+3$. Here $v$ is an equitable isolate and vertices $v_{4 i+1}, u_{4 j+3}, u_{n}$ are not equitably adjacent to each other. Therefore $D$ is an equitable independent set. Also $N^{e}(v) \cup N^{e}\left(v_{4 i+1}\right) \cup N^{e}\left(u_{4 j+3}\right) \cup N^{e}\left(u_{n}\right)=V\left(C H_{n}\right)$, where $0 \leq i \leq$ 
$\left\lfloor\frac{n}{4}\right\rfloor, 0 \leq j<\left\lfloor\frac{n}{4}\right\rfloor$. Therefore $D$ is an equitable dominating set. Hence $D$ is an equi independent equitable dominating set and $i^{e}\left(\mathrm{CH}_{n}\right)=\gamma^{e}\left(\mathrm{CH}_{n}\right)=$ $2\left\lfloor\frac{n}{4}\right\rfloor+3$.

Case 4: $n \equiv 3(\bmod 4)$

Here $N^{e}\left(v_{4 i+1}\right)=\left\{v_{4 i}, v_{4 i+2}, u_{4 i+1}\right\}$ and $N^{e}\left(u_{4 i+3}\right)=\left\{u_{4 i+2}, u_{4 i}, v_{4 i+3}\right\}$. Let $D=\left\{v, v_{4 i+1}, u_{4 i+3}\right\}$ where $0 \leq i \leq\left\lfloor\frac{n}{4}\right\rfloor$ with $|D|=2\left\lfloor\frac{n}{4}\right\rfloor+3$. Here $v$ is an equitable isolate and vertices $v_{4 i+1}, u_{4 i+3}$ are not equitably adjacent to each other. Therefore $D$ is an equitable independent set. Also $N^{e}(v) \cup N^{e}\left(v_{4 i+1}\right) \cup N^{e}\left(u_{4 i+3}\right)=V\left(C H_{n}\right)$, where $0 \leq i \leq\left\lfloor\frac{n}{4}\right\rfloor$. Therefore $D$ is an equitable dominating set. Thus $D$ is an equi independent equitable dominating set and $i^{e}\left(\mathrm{CH}_{n}\right)=\gamma^{e}\left(\mathrm{CH}_{n}\right)=2\left\lfloor\frac{n}{4}\right\rfloor+3$.

Hence

$i^{e}\left(C H_{n}\right)= \begin{cases}\gamma^{e}\left(C H_{n}\right)= \begin{cases}2\left\lfloor\frac{n}{4}\right\rfloor+3 & \text { for } n \equiv 2,3(\bmod 4), n \not \equiv 3 \\ \frac{n}{2}+1 & \text { for } n \equiv 0(\bmod 4), n \neq \equiv 4\end{cases} \\ \gamma^{e}\left(C H_{n}\right)+1=\left(2\left\lfloor\frac{n}{4}\right\rfloor+2\right)+1 \text { for } n \equiv 1(\bmod 4), n \not \equiv 5\end{cases}$

Illustration 2.13. The closed helm graph $\mathrm{CH}_{9}$ and its $i^{e}$-set of $\mathrm{CH}_{9}$ is shown by grey colored vertices in Figure 2.

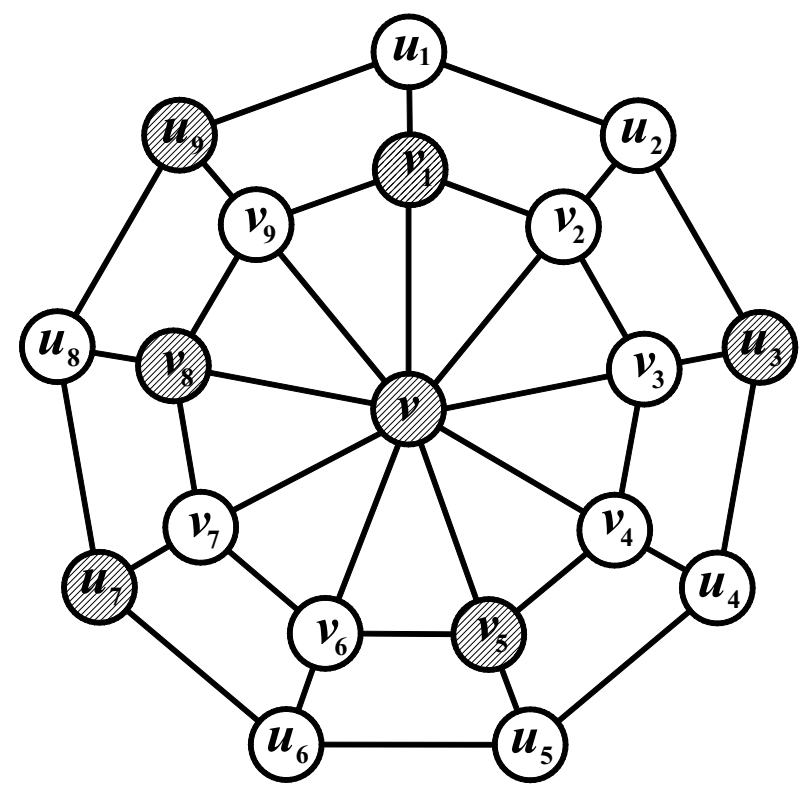

Figure 2: The closed helm graph $\mathrm{CH}_{9}$ and its $i^{e}$-set of $\mathrm{CH}_{9}$ 
Theorem 2.14. $i^{e}\left(W b_{n}\right)=i^{e}\left(C H_{n}\right)+n$.

Proof. Let $v_{1}, v_{2}, \ldots, v_{n}$ be the vertices of inner cycle, $u_{1}, u_{2}, \ldots, u_{n}$ be the vertices of outer cycle, $w_{1}, w_{2}, \ldots, w_{n}$ be the pendant vertices and $v$ be a apex vertex of web $W b_{n}$. Observe that pendant vertices are $w_{1}, w_{2}, \ldots, w_{n}$ are equitable isolates. There fore they must belongs to any equitable dominating set of web $W b_{n}$. Which implies that $i^{e}\left(W b_{n}\right) \geq i^{e}\left(C H_{n}\right)+n$.

Let $S$ be the $i^{e}$-set of $C H_{n}$ and $D=S \cup\left\{w_{1}, w_{2}, \ldots, w_{n}\right\}$ with $|D|=$ $i^{e}\left(\mathrm{CH}_{n}\right)+n$. We claim that $D$ is an equi independent equitable dominating set of web $W b_{n}$. Since pendant vertices $w_{1}, w_{2}, \ldots, w_{n}$ are equitable non adjacent to vertices $u_{1}, u_{2}, \ldots, u_{n}$ of outer cycle and set $S$ is an equitable independent set of $\mathrm{CH}_{n}$. Therefore $D$ is equitable independent set of web $W b_{n}$. While $D$ is equitable dominating set of web $W b_{n}$ as pendant vertices $w_{1}, w_{2}, \ldots, w_{n}$ are in $D$ and remaining vertices equitably dominated by set $S$. Therefore $D$ is an equi independent equitable dominating set of web $W b_{n}$ with $|D|=i^{e}\left(C H_{n}\right)+n$. Hence $i^{e}\left(W b_{n}\right)=i^{e}\left(C H_{n}\right)+n$.

Theorem 2.15. For any graph $G,\left\lceil\frac{n}{1+\Delta^{e}(G)}\right\rceil \leq \gamma^{e}(G) \leq n-\Delta^{e}(G)$.

Proof. Let $D$ be a $\gamma^{e}$-set of $G$. Each vertex of $G$ can dominate at most itself and $\Delta^{e}(G)$ other vertices. Hence $\gamma^{e}(G) \geq\left\lceil\frac{n}{1+\Delta^{e}(G)}\right\rceil$.

Let $v$ be a vertex of maximum equitable degree $\Delta^{e}(G)$. Then $v$ dominate $N^{e}[v]$ and the vertices in $V(G)-N^{e}[v]$ dominate them self. Hence $V(G)-$ $N^{e}[v]$ is dominating set of cardinality $n-\Delta^{e}(G)$. Which implies that $\gamma^{e}(G) \leq n-\Delta^{e}(G)$.

Remark 2.16. Cycle $C_{n}$ achieve the lower bound and Wheel graph $W_{4}$ achieve the upper bound of above Theorem.

Theorem 2.17. For any graph $G, i^{e}(G) \geq\left\lceil\frac{n}{1+\Delta^{e}(G)}\right\rceil$.

Theorem 2.18. $i^{e}(G) \leq n-\Delta^{e}(G)$.

Proof. Let $G$ be a graph. Any maximal equitable independent set of $G$ containing a vertex of maximum equitable degree $\Delta^{e}(G)$ contains at most $n-\Delta^{e}(G)$ vertices. Hence $i^{e}(G) \leq n-\Delta^{e}(G)$. 


\section{Concluding Remarks}

A new domination model has been introduced. We establish several general results in the context of newly defined concept. We also establish bounds for the equi independent equitable domination number as well as equitable domination number and also investigate graphs achieving these bounds.

\section{Acknowledgement}

The authors are highly thankful to the anonymous referees for their kind comments and fruitful suggestions on the first draft of this paper.

\section{References}

[1] C. Berge, Theory of Graphs and its Applications, Methuen, London, (1962).

[2] E. J. Cockayne and S.T. Hedetniemi, Independence graphs, Congr. Numer., X, pp. 471-491, (1974).

[3] E.J. Cockayne and S.T. Hedetniemi, Towards a theory of domination in graphs, Networks, 7, pp. 247-261, (1977).

[4] W. Goddard and M. A. Henning, Independent domination in graphs: A survey and recent results, Discrete Mathematics, 313, pp. 839-854, (2013).

[5] T. W. Haynes, S. T. Hedetniemi, and P. J. Slater, Fundamentals of domination in graphs, Marcel Dekker, New York, (1998).

[6] O. Ore, Theory of graphs, Amer. Math. Soc. Transl. 38, pp. 206-212, (1962).

[7] V.Swaminathan and K. Dharmalingam, Degree equitable domination on graphs, Kragujevac Journal of Mathematics, 35(1), pp. 191-197, (2011).

[8] S. K. Vaidya and N. J. Kothari, Some Results on Equi Independent Equitable Dominating Sets in Graphs, Journal of Scientific Research, 7(3), pp. 77-85, (2015). 
[9] S. K. Vaidya and N. J. Kothari, On equi independent equitable dominating sets in graphs, International Journal of Mathematics and Soft Computing, 6 (1), pp. 133-142, (2016).

[10] S. K. Vaidya and N. J. Kothari, Equi independent equitable domination number of cycle and bistar related graphs, IOSR Journal of Mathematics, 11 (6), pp. 26-32, (2015).

[11] D. B.West, Introduction to graph theory, 2nd ed., Prentice-Hall, New Delhi, India, (2003).

\section{S. K. Vaidya}

Department of Mathematics

Saurashtra University

Rajkot - 360005

Gujarat

India

e-mail : samirkvaidya@yahoo.co.in

and

\section{N. J. Kothari}

L. E. College

Sama Kathe,

Morbi-363642

Gujarat

India

e-mail : nirang_kothari@yahoo.com 